\title{
Association Mapping for Important Agronomic Traits in Core Collection of Rice (Oryza sativa L.) with SSR Markers
}

\author{
Ritu R. Saxena ${ }^{1}$, Sujit Roy ${ }^{*}$, Suman Rawte ${ }^{1}$, Parmeshwar K. Sahu ${ }^{1}$, \\ Ravi R. Saxena ${ }^{2}$ and S. B. Verulkar ${ }^{3}$ \\ ${ }^{1}$ Department of Genetics and Plant Breeding, ${ }^{2}$ Department of Agriculture Statistics and \\ Social Sciences (L), ${ }^{3}$ Department of Plant Molecular Biology and Biotechnology \\ Indira Gandhi Krishi Vishwavidyalaya, Raipur 492 012, Chhattisgarh, India \\ *Corresponding author
}

\section{A B S T R A C T}

\section{Keywords}

Rice landraces, Cultivated rice,

Linear model,

Rice breeding

Article Info

Accepted:

30 March 2020

Available Online:

10 April 2020
Mining elite genes within rice landraces is of importance for the improvement of cultivated rice. An association mapping for 12 agronomic traits was carried out using a core collection of rice consisting of 100 landraces (Panel 1) with 44 simple sequence repeat (SSR) markers. Our results showed that (1) 76significant (P, 0.05) trait-marker associations were detected using mixed linear model (MLM) within Panel 1 in two years, among which $32 \%$ were identical with previously mapped QTLs, and 11 significant associations had.10\% explained ratio of genetic variation; (2) A total of seven aforementioned trait-marker associations were verified within Panel 2 and 3 when using a general linear model (GLM) and 55 SSR markers of the 76 significant trait-marker associations. However, nonsignificant trait-marker association was found to be identical within three panels when using the MLM model; (3) several desirable alleles of the loci which showed significant trait-marker associations were identified. The research provided important information for further mining these elite genes within rice landraces and using them for rice breeding.

\section{Introduction}

As a staple cereal crop, rice (Oryza sativa L.) feeds more than $50 \%$ of the world's population and is one of the most important components of human diet in many regions of the world. Thus, genetic improvement of rice for yield is important to the meet food demand of a growing global population. Rice landraces have a greater genetic diversity than elite cultivars (or commercial cultivars). Mining elite genes of rice landraces is of importance for the improvement of cultivated rice. Linkage mapping and association 
mapping based linkage disequilibrium (LD) are two main methods for locating genes or QTLs. The major limitations of linkage mapping are that only two alleles at any given locus can be studied in bi-parental crosses and a low mapping resolution, whereas association mapping promises to overcome the limitations of linkage mapping. Association mapping has been widely used in plant research since it was firstly reported in maize.

Population structure may cause false positives in association mapping. To overcome this problem, an approach using a mixed model was proposed for association mapping, which take both population structure $(\mathrm{Q})$ and kinship (K) into account for the reduction of false positives. However, false positive might not be absolutely avoided it required that the significant associations identified within one population should be verified in another population. In this study, an association mapping was performed for 12agronomic traits from the core collection assessed with 44SSR markers. The study aimed to (1) perform association mapping for 12 important agronomic traits in the core collection to identify desirable alleles of the loci which showed significant trait-marker associations for rice breeding.

\section{Materials and Methods}

\section{Plant material}

Hundred genotypes of rice were taken for this research. The information for each genotype is shown in Table 1.

\section{Phenotyping}

All of the germplasm were cultivated at the instructional farm of Indira Gandhi Agricultural University, Raipur, Chhattisgarh $\left(21^{\circ} 16^{\prime} \mathrm{N}\right.$ latitude and $81^{\circ} 36^{\prime}$ E longitude with an altitude of $(289.60 \mathrm{~m}))$, during the late season (July-November) in wet season (Kharif) 2018. The highest temperature was $34.07^{\circ} \mathrm{C} \&$ lowest $27.9^{\circ} \mathrm{C}$ during the crop development time. The overall average rainfall during crop growing period was $(138.69 \mathrm{~mm})$. The highest rainfall received during August month was (39.29 mm).

Nurseries were raised and 21 days old seedlings were eventually transplanted in the field. The space between rows and between plants was set to $25 \mathrm{~cm}$ and $15 \mathrm{~cm}$, respectively. Thirty plants of each variety were grown in three rows with 10 plants per row. For each block, the five plants in the middle position of the second row of each variety were selected so that the marginal effect was avoided. 12 agronomic traits for these plants were investigated. A randomized complete block design with three replications was used during each season. The space between rows and between plants was set to 20 and $16.5 \mathrm{~cm}$, respectively. Thirty plants of each variety were grown in three rows with 10 plants per row. For each block, the five plants in the middle position of the second row of each variety were selected so that the marginal effect was avoided. 12 agronomic traits for these plants were investigated. Heading date (HD) was recorded as days from sowing to flowering time when $50 \%$ of the individuals of one variety started flowering. Plant height $(\mathrm{PH})$, panicle length (PL), flag leaf length (FLL), and flag leaf width (FLW) were measured in centimetres. Number of effective tillers (NT) was counted as effective tillers. For1000-grain weight (1000GW), 100 grains were measured in grams with three replicates and then its average was multiplied by 10 . Number of filled grains (NFG) was counted. Biological yield (BY) was recorded as the weight of each of the 5 sun dried plant except root was recorded in grams. The grain (filled) yield (GY) of each of the 5 plants was recorded in $(\mathrm{g})$ after sun drying for five to 
eight days after harvesting and averaged. Harvest index (HI) was estimated as the ratio of grain yield to biological yield and the ratio of grain yield and biological yield are expressed in $\%$.

\section{Genotyping}

44 SSR markers evenly distributed across the 12 chromosomes. Rice genomic DNA was extracted out from each of the landraces of rice using $\mathrm{CTAB}$ method. DNA samples isolated from each line were quantified on Nano Drop Spectroscopy (NANODROP, 2000c) and the final concentration of DNA was $50 \eta \mathrm{g} / \mu \mathrm{l}$ for PCR analysis. The volume of the polymerase chain reaction (PCR) was $10 \mu 1$ in Axygen make 96 well PCR plates. The profile of the PCR program was as follows: $94^{\circ} \mathrm{C}$ for $5 \mathrm{~min}$ followed by 29 cycles of $94^{\circ} \mathrm{uC}$ for $1 \mathrm{~min}, 55^{\circ} \mathrm{C}$ for $1 \mathrm{~min}$, $72^{\circ} \mathrm{C}$ for 1 min with a final extension of 5 minutes at $72^{\circ} \mathrm{C}$. PCR products were separated in size by $56 \%$ polyacrylamide gel electrophoresis and detected by ethidium bromide. The size of PCR products were detected by BIORAD gel doc XR + System. The length of each allele was compared to the standard bands of the standard marker and scored.

\section{Data analysis}

Means and standard deviation (SD) for 12 traits were calculated using MS Excel software. The percentage of phenotypic variation explained by population structure was calculated using a General Linear Model (MLM) with software SPSS 17.0 for Windows (SPSS Inc. Chicago, IL, USA). The broad-sense heritability $\left(\mathrm{H}^{2}\right)$ was calculated asH ${ }^{2}=\sigma_{\mathrm{g}}^{2} /\left(\sigma_{\mathrm{g}}^{2}+\sigma_{\mathrm{e}}^{2}\right)$, where $\sigma_{\mathrm{g}}^{2}$ is the genetic variance, $\sigma_{\mathrm{e}}^{2}$ is the environmental variance and correlation coefficients between traits were calculated using the software SPSS. Polymorphism information content (PIC) which measures the extent of polymorphism for marker gene(s) or marker sequence(s)was calculated using the program POWERMARKER V3.25. Software Structure V2.3.1 was used to infer population structure and get $\mathrm{Q}$ matrices. During the running, a range of genetic clusters from $\mathrm{K}=1$ to 15 with the admixture model was examined, and for each $\mathrm{K}$ it was replicated 5 times. Each run implemented with a burn-in period of 100,000 steps followed by 100,000 MonteCarlo Markov Chain replicates.

Due to the distribution of $\mathrm{L}(\mathrm{K})$ did not show a clear cut off point for the true $\mathrm{K}$, an adhoc measured was used to detect the numbers of subgroup. That runs with the maximum likelihood was applied to subdivide the varieties into different subgroups based on the maximum membership probability. A Qmatrix was obtained from the membership probability of each variety.

The Q-matrix was used for further association mapping. Quantile-quantile plots were generated for observed against expected $2 \log 10(\mathrm{P})$ where observed $\mathrm{P}$ values were obtained from association mapping and expected $\mathrm{P}$ values from the assumption that no associations happened between marker and trait. Association analysis was performed using the software TASSEL (www.maizegenetics.net/tassel). For the mixed linear model (MLM) method, both $\mathrm{K}$ and $\mathrm{Q}$ matrices were incorporated, whereas for the GLM method, only population structure information (Q-matrix) was used as a covariate.

Significance of associations between loci and traits were determined by their Pvalues $(\mathrm{P}$, $0.05)$ which were calculated by the statistical models, and the phenotypic variance explained by the significant loci was calculated through analysis of variance (ANOVA). Since MLM method performs 
better in controlling spurious associations than GLM method, we first ranked the significant $(\mathrm{P}, 0.05)$ association from MLM and then compared the significance of these markers $(\mathrm{P}, 0.05)$ in the permutation based on GLM association tests.

\section{Results and Discussion}

\section{Phenotypic data}

The range for days to $50 \%$ flowering varied from 75.00 days (R-RF 75) to 118.00 days (Danwar) with the overall mean of 96.15 days. Four categories for days to $50 \%$ flowering (table 4.4) were reported i.e. early (91- 90days), medium (91-110days), late (111-130days) and very late (> 131days). The coefficient of variation was $10.92 \%$. The range for plant height varied from $75.01 \mathrm{~cm}$ (Bagri) to $271.45 \mathrm{~cm}$ (Hanuman Langur) with the overall mean of $130.49 \mathrm{~cm}$. The coefficient of variation of plant height was $19.74 \%$.The range for leaf length varied from $22.30 \mathrm{~cm}$ (Bisni-I) to $76.61 \mathrm{~cm}$ (Roti) with the overall mean of $47.55 \mathrm{~cm}$.

The coefficient of variation was $29.42 \%$.The range for leaf width varied from $0.78 \mathrm{~cm}$ (JeeraPhool) to $2.01 \mathrm{~cm}$ (R-RF 75) with the overall mean of $1.26 \mathrm{~cm}$. For flag leaf width, the coefficient of variation was $16.80 \%$. It ranged from $2.10 \mathrm{~cm}$ (HathiPanjara) to $13.40 \mathrm{~cm}$ (Bagri) with the overall mean of 5.83. The coefficient of variation was $35.15 \%$.

Four category of panicle length was found i.e. Very short $(<16 \mathrm{~cm})$, Short $(16-20 \mathrm{~cm})$, Medium $(21-25 \mathrm{~cm})$ and Long $(26-30 \mathrm{~cm})$ (Table 4.5). The maximum and minimum panicle length were found $29.67 \mathrm{~cm}$ (Dokraemechha) and $18.51 \mathrm{~cm} \mathrm{(Koto),}$ respectively, with the overall mean of 24.65 $\mathrm{cm}$. Highest panicle length was found 29.67 $\mathrm{cm}$ in Dokraemechha followed by $29.05 \mathrm{~cm}$ in BhatanPhool-I and $28.84 \mathrm{~cm}$ in Shyam Jira - I and $28.62 \mathrm{~cm}$ in BhainsaPunchhi. The coefficient of variation was $10.09 \%$. 1000grain weight ranged from $7.70 \mathrm{~g}$ to $43.45 \mathrm{~g}$ with an average weight of $22.31 \mathrm{~g}$. The maximum 1000-seed weight recorded in Dokra-Dokri (43.45 g) followed by HathiPanjara (42.31 g) and Chiko (40.96 g). The minimum 1000-seed weight recorded in Ram Jira (7.70 g).

The coefficient of variation was $37.98 \%$. Number of filled grains ranged from 25.45 to 531.45 with an average of 141.18. The maximum 1000-seed weight recorded in JouPhool (531.45) followed by Ram Jira (381.12). The minimum 1000-seed weight recorded in Bhejari (25.45). The coefficient of variation was $60.40 \%$. Number of unfilled grains ranged from 9.74 to 220.00 with an average of 33.61. The maximum 1000-seed weight recorded in Bhejari (220.00). The minimum 1000-seed weight recorded in Koto (9.73). The coefficient of variation was $85.20 \%$.

Total number of grains ranged from 57.42 to 606.49 with an average weight of 174.80 . The maximum 1000-seed weight recorded in JouPhool (606.49). The minimum 1000-seed weight recorded in Koto (57.42). The coefficient of variation was $32.01 \%$. The mean performance of biological yield was $295.63 \mathrm{~g}$. It showed variation from 72.08 to $774.04 \mathrm{~g}$. The maximum biological yield was recorded in Lanji (774.04 g) followed by SindorSenga (654.88 g) and the minimum were recorded in Luchai-A (72.08 g).

The coefficient of variation was $13.63 \%$. Harvest index was found to vary from $1.27 \%$ to $18.72 \%$ with an overall mean of $6.37 \%$. The maximum harvest index was recorded in PadariDhan (18.72\%). The minimum harvest index was recorded in Koto $(1.27 \%)$. The coefficient of variation was $43.22 \%$. The range 
for grain yield (g) varied from $4.07 \mathrm{~g}$ to 29.66 $\mathrm{g}$ with a mean value of $15.66 \mathrm{~g}$. The highest grain yield $(\mathrm{g})$ was recorded in BailaAankhi (29.66 g) followed by SuaPankhi (29.60 g) and BawatiChudi (29.19 g). The minimum grain yield $(\mathrm{g})$ was recorded in Koto $(4.07 \mathrm{~g})$. The coefficient of variation was $37.24 \%$.

Heritability estimates provide the information regarding the amount of transmissible genetic variation to total variation and determine genetic improvement and response to selection. Thus, heritability is the heritable portion of the phenotypic variance. It is a good index of the transformation of characters from parents to their offspring (Falconer, 1981). Heritability and genetic advance are important selection parameters. Heritability estimates along with genetic advance are normally more helpful in predicting the grain under selection than heritability estimates alone.

Improvement in the mean genotypic value of selected plants over the parental population is known as genetic advance. It is the measure of genetic gain under selection. The success of genetic advance under selection depends on genetic variability, heritability and selection intensity. In the present investigation heritability in broad sense and genetic advance were calculated for 33 yield and quality characters under study and is presented in Table4.3.

Highest estimate of heritability was found for biological yield (99.47\%) followed by days to flowering (98.69\%), grain yield $(98.09 \%)$, thousand grain weight $(98.63 \%)$, harvest index (97.66\%), flag leaf width (95.34\%), flag leaf length $(89.35 \%)$ and total number of grains $(62.91 \%)$. Rest of the traits was found to have moderate heritability. It clearly indicates that most likely the heritability is due to additive gene effects and selection may be effective. These findings are in agreement with findings of Choudhary et al., 2004; Satheeshkumar and Saravanan, 2012; Sravan et al., 2012 and Khare et al., 2016.

\section{Molecular characterization}

Genetic associations among 100 accessions were analysed, based on phenotypic variation of yield traits with the help of 44 SSR markers covering all the chromosomes. A total of 217 alleles were amplified and the number of alleles per locus generated by each marker ranged from 3 to 11 alleles with an average number of 4.93 alleles per locus. Maximum number of alleles (11) was amplified by marker RM 164 marker. The PIC value across markers ranged from 0.24 to 0.85 with an average of 0.66 . Maximum PIC value was observed on chromosome 1 (RM $164=0.85$ ) followed by RM 248 of chromosome 7 (0.84) and RM 474 of chromosome 1 (0.82).

\section{Population structure analysis}

The Bayesion model-based Structure v2.3.4 program was used to infer population structure of rice genotypes. The 100 lines were divided into two sub groups (Fig 4.4 and 4.5) based on the result of Structure Harvester, as delta K kinship was highest at $\mathrm{K}=2$. With population inferred ancestry $(\mathrm{Q})=$ 0.80, 48 lines were assigned to subgroup POP1, rest 48 lines were assigned to subgroup POP2 and four lines namely, R RF 75 (49); KadamPhool (50); AmaJhopa (51) and KoudiDhull (52) were assigned to admixture (AD) which has less than $<0.80$ inferred ancestry (Fig 4.5). Courtois et al., (2012) has successfully detected two subgroups in their study population and assigned rice varieties into two groups with few admixture lines. Our results are also in conformity with the findings of Borba (2010) suggesting that using structure analysis, the accessions were sub divided into two panels. Likewise, the 
association of yield traits with SSR markers was undertaken with MLM model, with markers and sub population as fixed factors, and kinship matrix as random factor.

\section{Marker-trait association}

Association analysis between SSR markers and thirteen agronomic and yield attributing traits was carried out using MLM model over the 100 rice germplasm lines. Eleven SSR markers were found to be tightly linked with the panicle length trait. These markers covered the entire linkage groups except chromosome \# 2, 5, 7, 8 and 11.

Apart from this, 4 markers each for days to flowering followed by, plant height and total number of grains, 7 markers for flag leaf length, 5 markers both for flag leaf width and biological yield, 3 markers for number of tillers, harvest index and thousand grain weight, 6 markers for number of filled grains and 2 markers each for number of unfilled grains and grain yield were found to be significantly association with the traits at 0.05 threshold level of significance. However, these were significantly associated with all the yield traits (Table 4.11) at the FDR correction level in the entire population. For days to flowering, lowest phenotypic variation $(17.08 \%)$ by RM 208 whereas highest phenotypic variation was explained by marker RM $338(22.68 \%)$ followed by RM $307(21.05 \%)$ and RM 287 (18.89 \%) which was significantly associated with the trait.

Lowest phenotypic variation in plant height was observed by RM 212 of C\# 1 (12.07\%) whereas highest variance was seen in C\# 3 with RM 338 (23.94 \%) followed by RM 105 (C\#9) and RM 348 (C\#4) (28.92\%).The total phenotypic variation for flag leaf length was observed lowest in RM $105(8.31 \%)$ and highest in RM 431 (18.63\%) whereas in case of flag leaf width, it ranged from RM 235
(20.18\%) to RM 247 (32.58\%).RM338 of C\#3 (11.05\%) exhibited lowest and RM 348 (24.89\%) showed highest followed by RM $510(22.14 \%)$ for number of tillers and RM 105 of C\# 9 (10.96 \%) lowest and RM 269 (22.89\%) phenotypic variation in panicle length. There were two markers, namely RM 283 and 431 from linkage group 1, RM 55 and RM 338 from linkage group 3, RM 124 and RM 307 from C\# 4, RM 553 and RM 105 of linkage group 9 and RM 269 (C\#10) and RM 235 (C\#12) were found to have tight linkage with the trait i.e., panicle length.

Five SSR markers namely, RM 133 (C\#6), RM 536 (C\#11), RM 312 (C\#1), RM 474 (C\#10) and RM 11 (C\#7) were to have tight association with biological yield, the phenotypic variance ranging from $4.2 \%$ (RM 11) to $25.68 \%$ (RM 133).For grain yield two primers, RM 208 (C\#2) and RM 247 (C\#12) and for harvest index, RM 133 (C\#6), RM 208 (C\#2) and RM 271 (C\#10) were found to be significantly associated with the above traits.

Three markers viz., RM 474, RM 247 and RM 316 were significantly associated with thousand grain weight, likewise, six markers showed tightly linked response with number of filled grains. Similarly, RM 316 and RM 433 for number of unfilled grains and four markers, RM 474, RM 413, RM 536 and RM 248 showed significant and tight linkage with total number of grains.

Agrama et al., 2006 conveyed that to make advances in rice breeding it is essential to understand the relatedness and ancestry of introduced rice accessions and identify SSR markers associated with agronomically important phenotypic traits, for example yield. Identification of a candidate gene for panicle length in trice through association mapping is an important trait for improving panicle architecture and grain yield in rice. 
Using the MLM model, RM 338 of chromosome \#3 was found to have significant association with days to flowering, plant height, flag leaf width, number of tillers and panicle length.RM 208 of linkage group 2 showed relationship with days to flowering, grain yield and harvest index. For grain yield, thousand grain weight and number of filled grains and flag leaf width RM 247 of chromosome 12 showed significant association and RM 133 (C\#6) for biological yield, harvest index and panicle length.

Similar results were reported by Liu et al., 2016. The research provided important information for further mining these elite genes within rice landraces and using them for rice breeding. RM 287 and RM 447 were found to have significant association with flag leaf length and thousand grain weights, respectively. Zhang et al., 2014 also reported same marker with the above traits. In our material, panicle length showed association with RM 283 of chromosome 1, Rabaei et al., 2015 reported a QTL named $q P L-1$ responsible of panicle length between flanking markers RM 283 -RM 8132. Likewise another QTL for panicle length was found on C\#4 named qp14.1 between flanking markers RM 131-RM 124 as reported by Jia et al., 2019. In our germplasm accessions, RM 124 also showed significant association with the trait.

The results have clearly shown that structures association mapping in one of the feasible options to identify major effect QTLs for yield traits in rice. These marker-trait associations could be further validated and used in marker assisted breeding for improving particular trait in any rice variety and can be further confirmed in new set of population as well as in bi-parental mapping population.

Table.1 List of germplasm accessions

\begin{tabular}{|l|l|l|l|l|l|l|l|}
\hline S. No. & Name & S. No. & Name & S. No. & Name & S. No. & Name \\
\hline $\mathbf{1}$ & Bagri & 26 & Kanak & 51 & Amajhopa & 76 & Ram Jira \\
\hline $\mathbf{2}$ & Hardichudi & 27 & Mehapal & 52 & Koudidhull & 77 & Bhejari \\
\hline $\mathbf{3}$ & Koto & 28 & TebarooMundaria & 53 & Saupankhi & 78 & Danwar \\
\hline $\mathbf{4}$ & Kotte (II) & 29 & Padaridhan IV & 54 & DokraDokri & 79 & Karhani \\
\hline $\mathbf{5}$ & Sathadhan & 30 & Budhiyawako & 55 & Parmal & 80 & Chiko \\
\hline $\mathbf{6}$ & Karhani & 31 & BD kankaribija & 56 & Dokraemechha & 81 & FarsaPhool \\
\hline $\mathbf{7}$ & Kohaka & 32 & Bawatichudi & 57 & Roti & 82 & BailaAankhi \\
\hline $\mathbf{8}$ & Luchai(A) & 33 & Kalajira & 58 & Khatiapati & 83 & Bokra Mundi \\
\hline $\mathbf{9}$ & AngurGuchcha & 34 & Sonapan & 59 & Hathipanjra & 84 & HunumanLangur \\
\hline $\mathbf{1 0}$ & Basigal(ii) & 35 & Bakal & 60 & CR-1014 & 85 & JalPonga \\
\hline $\mathbf{1 1}$ & Bhejari & 36 & Cross 116 & 61 & Elayachi & 86 & Banda \\
\hline $\mathbf{1 2}$ & Bhulau & 37 & Deshilaldhan & 62 & Tulsimanjari & 87 & Lanji \\
\hline $\mathbf{1 3}$ & Bodi & 38 & IR 42253 & 63 & Shyam jira-1 & 88 & Raja Bangla \\
\hline $\mathbf{1 4}$ & PeeleeLuchai & 39 & Lalmati & 64 & LoktiMachhi & 89 & BhainsaMundariya \\
\hline $\mathbf{1 5}$ & TulsiPhool & 40 & Laloo-14 & 65 & Muni Bhog & 90 & NariyalChudi \\
\hline $\mathbf{1 6}$ & Silipat & 41 & Jhitpiti & 66 & JouPhool & 91 & Kating \\
\hline $\mathbf{1 7}$ & Unknown & 42 & WR99 & 67 & BhainsaPunchhi & 92 & Bhamasur \\
\hline $\mathbf{1 8}$ & AmaDhul & 43 & E-1702 & 68 & Bhanta Phool-1 & 93 & Paltu \\
\hline $\mathbf{1 9}$ & Baisur & 44 & Chaptigurmatia & 69 & Lahsun Bhog & 94 & Sindursenga \\
\hline $\mathbf{2 0}$ & Bylao & 45 & Elayachi & 70 & Ichchawati & 95 & Swarna \\
\hline $\mathbf{2 1}$ & AsamChudi & 46 & Bisni-I & 71 & Laxmi Bhog & 96 & MTU-1010 \\
\hline $\mathbf{2 2}$ & Bhaniya & 47 & Moroberekan & 72 & Tulsi Mala & 97 & IR64 \\
\hline $\mathbf{2 3}$ & FarsaPhool & 48 & Nagina-22 & 73 & Jou Phool-2 & 98 & R-RF-75 \\
\hline $\mathbf{2 4}$ & Jalle & 49 & R-RF-75 & 74 & JeeraPhool & 99 & IGKV R1 \\
\hline $\mathbf{2 5}$ & Kanak Jira & 50 & KadamPhool & 75 & TulsiMongra & 100 & Danteshwari \\
\hline & & & & & \\
\hline
\end{tabular}


Table.2 Descriptive statistics, percentage of phenotypic variation explained by population structure $\left(\mathrm{R}^{2}\right)$, and heritability in broad sense $\left(\mathrm{h}^{2}\right)$ for 12 agronomic traits

\begin{tabular}{|l|l|l|l|l|l|}
\hline Genetic Parameters & Mean \pm SD & Range & $\left.\mathbf{h}^{\mathbf{2}} \mathbf{\%}\right)$ & $\mathbf{R}^{\mathbf{2}}$ & $\mathbf{C V}(\mathbf{\%})$ \\
\hline DTF (Days) & $96.15 \pm 10.50$ & $75.00-118.00$ & 98.69 & 10.92 \\
\hline PH $(\mathbf{c m})$ & $130.49 \pm 25.76$ & $75.01-271.45$ & 47.98 & 19.74 \\
\hline FLL $(\mathbf{c m})$ & $47.55 \pm 13.99$ & $22.30-76.61$ & 89.35 & 29.42 \\
\hline FLW $(\mathbf{c m})$ & $1.26 \pm 0.21$ & $0.78-2.01$ & 95.34 & 16.80 \\
\hline NT & $5.83 \pm 2.05$ & $2.10-13.40$ & 91.98 & 35.15 \\
\hline PL (cm) & $24.65 \pm 2.49$ & $18.31-29.67$ & 47.99 & 10.09 \\
\hline TGW (g) & $22.31 \pm 8.47$ & $7.70-43.45$ & 98.63 & 37.98 \\
\hline NFG & $141.18 \pm 85.27$ & $25.45-531.45$ & 60.29 & 60.40 \\
\hline NUFG & $33.61 \pm 28.64$ & $9.73-220.00$ & 46.60 & 85.20 \\
\hline TNG & $174.80 \pm 55.97$ & $57.42-606.49$ & 62.91 & 32.01 \\
\hline BYP (g) & $295.63 \pm 40.31$ & $72.08-774.04$ & 99.47 & 13.63 \\
\hline HI $\mathbf{( \% )}$ & $6.37 \pm 2.61$ & $1.27-18.72$ & 97.66 & 43.22 \\
\hline GYP (g) & $15.66 \pm 5.83$ & $4.07-29.66$ & 98.09 & 37.24 \\
\hline
\end{tabular}

The 100 lines were divided into two sub groups based on the result of Structure Harvester, as delta K kinship was highest at $\mathrm{K}=2$. With population inferred ancestry $(\mathrm{Q})=$ $0.80,48$ lines were assigned to subgroup POP1, rest 48 lines were assigned to subgroup POP2 and four lines namely, R RF 75 (49); KadamPhool (50); AmaJhopa (51) and KoudiDhull (52) were assigned to admixture (AD) which has less than $<0.80$ inferred ancestry. Eleven SSR markers were found to be tightly linked with the panicle length trait. These markers covered the entire linkage groups except chromosome \# 2, 5, 7, 8 and11. Using the MLM model, for grain yield RM 208 and RM 247 were found to be associated for the trait.

\section{References}

Agrama, H. A., Eizenga, G. C., and Yan, W. 2006. Association mapping of yield and its components in rice cultivars. Mol. Breeding 19, 341-356.

Borba TC, Brondani RV, Breseghello F, Coelho ASG, Mendonça JA, Rangel PHN, Brondani C. 2010. Association mapping for yield and grain quality traits in rice (Oryza sativa L.). Genet
MolBiol 33(3):515-524.

Choudhary, M., Sarawgi, A.K. and Motiramani, N.K. 2004. Genetic variability of quality, yield and yield attributing traits in aromatic rice (Oryza sativa L.). Adv. in Plant Sci., 17 (2): 485-490.

Courtois B, Audebert A, Dardou A, Roques S, Ghneim-Herrera T (2013) Genomewide association mapping of root traits in a japonica rice panel. PLoS One 8(11):78037.

Jia L, Yan W, Zhu C, Agrama H A, Jackson A, Yeater K, Li X, Huang B, Hu B, McClung A, Wu D. 2019. Allelic analysis of sheath blight resistance with association mapping in rice. PLOS ONE, 7, e32703.

Khare, R., Singh, K.S., Singh, A., Singh. P.K. 2016. Genetic variability, association and diversity analysis in upland Rice (Oryza sativa L).SAARC Journal of Agriculture, 12(2), 40-51.

Liu, E., Liu, Y., Wu, G., Zeng, S., Thi, T.G.T., Liang, L., Liang, Y., Dong, Z., She, D., Wang, H. and Zaid, I. U. 2016. Identification of a candidate gene for panicle length in rice (Oryza sativa L.) via association and linkage analysis. 
Front. Plant. Sci., 7(6), 341-352.

Satheeshkumar, P. and Saravanan, K. 2012. Genetic variability, correlation and path analysis in rice (Oryza sativa L.). Int. J. Curr. Res. 4(9): 82-85.
Sravan, T., Rangare, N. R., Suresh, B. G., and Kumar, S. R. 2012. Genetic variability and character association in rainfed upland rice (Oryza sativa L). Journal Rice Research, 5(1\&2), 24-28.

\section{How to cite this article:}

Ritu R. Saxena, Sujit Roy, Suman Rawte, Parmeshwar K. Sahu, Ravi R. Saxena and Verulkar, S. B. 2020. Association Mapping for Important Agronomic Traits in Core Collection of Rice (Oryza sativa L.) with SSR Markers. Int.J.Curr.Microbiol.App.Sci. 9(04): 3216-3224.

doi: https://doi.org/10.20546/ijcmas.2020.904.374 